\title{
Initial Assessment of Electron and X-Ray Production and Charge Exchange in the NDCX-II Accelerator
}

\author{
by \\ R.H. Cohen \\ Lawrence Livermore National Laboratory
}

\author{
HIFS-VNL \\ Lawrence Berkeley National Laboratory (on behalf of U.S. HIFS-VNL) \\ 1 Cyclotron Road, Berkeley, CA 94720
}

\author{
Accelerator Fusion Research Division \\ Ernest Orlando Lawrence Berkeley National Laboratory \\ University of California \\ Berkeley, California 94720
}

February 2010

This document was prepared as an account of work sponsored by the United States Government. While this document is believed to contain correct information, neither the United States Government nor any agency thereof, nor The Regents of the University of California, nor any of their employees, makes any warranty, express or implied, or assumes any legal responsibility for the accuracy, completeness, or usefulness of any information, apparatus, product, or process disclosed, or represents that its use would not infringe privately owned rights. Reference herein to any specific commercial product, process, or service by its trade name, trademark, manufacturer, or otherwise, does not necessarily constitute or imply its endorsement, recommendation, or favoring by the United States Government or any agency thereof, or The Regents of the University of California. The views and opinions of authors expressed herein do not necessarily state or reflect those of the United States Government or any agency thereof or The Regents of the University of California.

This work was supported by the Director, Office of Science, Office of Fusion Energy Sciences, of the U.S. Department of Energy under Contract No. DE-AC0205CH11231. 


\title{
Initial Assessment of Electron and X-Ray Production and Charge Exchange in the NDCX-II Accelerator
}

\author{
R.H. Cohen, LLNL and HIFS-VNL \\ Feb. 18, 2010
}

The purpose of this note is to provide initial assessments of some atomic physics effects for the accelerator section of NDCX-II. There are several effects we address: the production of electrons associated with loss of beam ions to the walls, the production of electrons associated with ionization of background gas, the possibly resultant production of X-rays when these electrons hit bounding surfaces, and charge exchange of beam ions on background gas. The results presented here are based on a number of caveats that will be stated below, which we will attempt to remove in the near future.

\section{Electron release from lost beam ions striking surrounding surfaces}

WARP simulations (by Dave Grote) show some loss of beam ions to surrounding surfaces as the beam transits the accelerator, so one might be concerned about electron desorption at these surfaces. This is a major source of concern in quadrupole-based accelerators, as magnetic field lines directly connect the bounding surfaces with the central region of the accelerator. NDCX-II has a strong solenoidal field, but there could be a concern if ions were lost at axial locations with a relatively weak magnetic field, as the field lines could provide a path for electrons toward the center of the accelerator up or down stream. However, examination of the WARP simulations show that ions are lost only in regions of appreciable magnetic field, such that the field lines and electron orbits return to the wall without making it far (radially) into the solenoid, or without traversing an accelerating gap. These electrons are confined to near the accelerator edge and so have negligible effect on the beam, and live for one or two transit times along a field line between wall intersection points before being reabsorbed; their probability of emitting a secondary electron is negligible.

It is conceivable that there could be some ion loss within a gap, which can result in a desorbed electron that is immediately accelerated by the gap field. The electrons born in that portion of a gap where the field lines move into the accelerator upstream of the gap will follow the field line and again hit the wall, and one might be concerned about the possibility of releasing a secondary electron upon that impact. Such an electron will have gained an energy comparable to the gap accelerating potential, up to about $250 \mathrm{KeV}$. Secondary electron yield for this energy range is well below 1 (see for example Ref. [1]). Furthermore, (assuming the secondary emission process to be local with regard to surface location) any secondaries emitted through this process will be on field lines which would take them inward away from the wall only downstream (relative to ion beam propagation) of their emission point; so these electrons quickly return to their birth location. Hence the only concern associated with ion loss in gaps is electrons accelerated through a single gap field (energy $\sim 250 \mathrm{keV}$ ), with a small degree of number amplification due to low secondary yield. Based on the WARP run examined, this seems to be a negligible concern, and in general is highly unlikely since there 


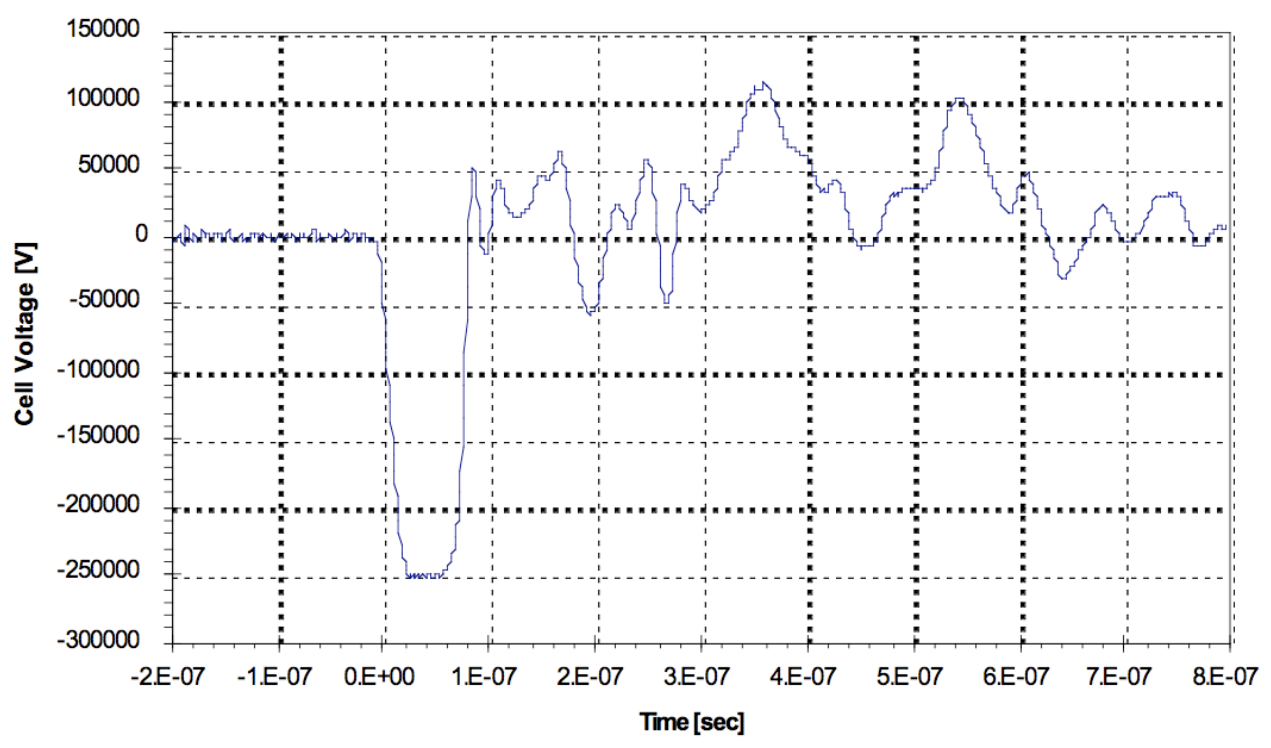

Figure 1: Waveform for an NCX-II accelerating module

is a break in the beam pipe in the gaps.

Another location where WARP shows significant beam loss is in the drift section, but as there is already a copious inventory of electrons in this region, associated with the neutralizing plasma, the electrons emitted from walls in this region are of no consequence other than possibly assisting in the neutralization.

\section{Electron production by gas ionization}

The ion beam impacting background gas will result in ionization of that gas, producing electrons on field lines which can traverse multiple gaps. Of concern is that such electrons might be accelerated upstream through a number of active gaps, acquire substantial energy, and then strike a surface. This concern is prompted by the observation that, while the gap accelerating schedule has at most two gaps on at once, the voltage response for a single gap is such that there is significant ringing after the gap is nominally turned off (see Fig. 1). We can quickly dispel this notion; the acceleration experienced by an electron is to a very good approximation restricted to that of two gaps. The logic for this statement is as follows.

For an electron which is accelerated upstream through a gap while fully on acquires an energy of about $250 \mathrm{keV}$. The (longitudinally directed) speed of such an electron is $v \sim$ $2.2 \times 10^{8} \mathrm{~m} / \mathrm{s}$. The time for such an electron to traverse the full $10 \mathrm{~m}$ length of the accelerator is about $5 \mathrm{~ns}$. This is effectively instantaneous on the timescale of the programming of the accelerator gaps (and of the ion beam transit).

If we assume that all gaps exhibit the after-pulse response shown in Fig. 1, then an electron which has traversed an accelerating gap will see accelerating potentials in the furtherupstream gaps given by a displacement to the right in FIg. 1 approximately by the amount of the timing delays of successive gap waveforms. That is, the electron is accelerated from 


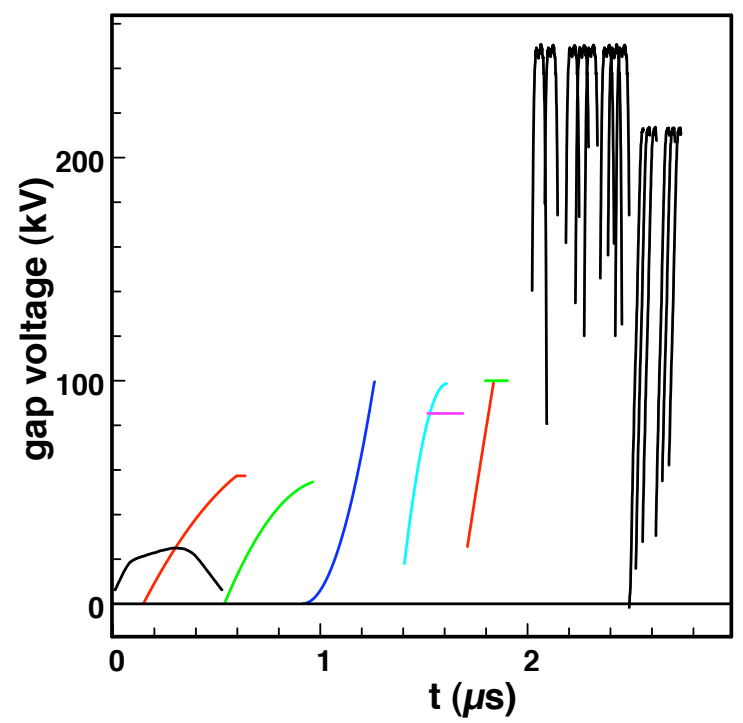

Figure 2: Ensemble of waveforms for NDCX-II, from Ref. [1]

an initial energy $\mathcal{E}_{\text {initial }}$ to an energy $\mathcal{E}_{\text {final }}$ given by

$$
\mathcal{E}_{\text {final }}=\mathcal{E}_{\text {initial }}+\sum_{i} V\left(t+\Delta \tau_{i}\right)
$$

where $t$ is the time when the electron traverses the first gap, relative to the start of that gap's pulse, and $\Delta \tau_{i}$ is the advance in time of the $i$ th pulse upstream from the first gap. This sum could be evaluated explicitly for a given electron given its birth position and time, and the various gap pulse schedules. Here it suffices to note that the gap pulse start times differ by an amount comparable to the gap "on" time, so any electron that has made it through two gaps will see a sequence of at most about 10 gaps with appreciable voltage, of which three or fewer are in a direction that adds to the main gap voltage. So the expectation is that the effect of the ringing will be to modestly decrease the electron energy.

Also, looking at a typical ensemble of waveforms (Fig. 2, from Alex Friedman's APS paper $[2]$ ), we see that there are no instances of more than one gap on at flat-top simultaneously, though there are a number of instances of one gap being at flat top while a neighbor is rising or falling. So the only electrons which could be accelerated as high as twice the maximum $(500 \mathrm{~V})$ are those that experience their initial acceleration just as a gap is first getting to full voltage. A much more typical situation is that an electron will gain the full potential of one gap and at most half of a second gap, around $375 \mathrm{KeV}$. Electrons born in the final set of tilt-applying gaps might see the effect of three simultaneously on gaps, but still only one at peak voltage, and the peak is only about $200 \mathrm{KV}$. So even for these $375 \mathrm{KeV}$ should represent a good upper bound.

Next we comment on the fraction of electrons that experience acceleration by the main gap pulse. This depends on the spectrum of the ionized electrons. An upper limit (and the basis of my initial approxmations) is to assume that all electrons see an accelerating 
gap. In actuality, electrons can be created anywhere along the accelerator, and if their typical energy is a few eV, only a small fraction of them will experience a gap that is fully accelerating for them. Roughly, if the mean thermal speed of electrons is $v_{t h}$, then a fraction $f_{p} \sim(1 / 4) v_{t h} \tau_{p u l s e} / L_{s o l}$ arrive at their first accelerating gap in time to experience significant acceleration, where $\tau_{\text {pulse }}$ is the length of a gap's main pulse and $L_{\text {sol }}$ is the inter-gap length spacing. The factor of $1 / 4$ accounts for the facts that half the electrons are born moving away from the accelerating gap, and that electrons born any time after the beginning of the pulse "on" period have less time than $\tau_{\text {pulse }}$ to get to the gap before its voltage is turned off. This reduction factor makes sense for electron energies less than that at which an electron can traverse the space between gaps during the pulse duration, which is about $100 \mathrm{eV}$ for a solenoid spacing of $20 \mathrm{~cm}$ and a pulse duration of $5 \times 10^{-8} \mathrm{sec}$. Since the ionized electrons will be mostly in the few ev range [3], this is reasonable. For $3 \mathrm{eV}$ electrons, we find a fraction $f \sim 0.045$. As a conservative upper limit we take our estimate for $10 \mathrm{eV}$ electrons, for which $f_{p}=0.083$. To be further conservative we round this up and take $f_{p}=0.1$.

There is an additional reduction factor associated with the bowing of field lines; electrons born far enough from the center of the beam pipe will follow field lines into the wall before they reach the gap. The fraction can be estimated as $f_{b}=\left(r_{g} / r_{b}\right)^{2}$, where $r_{g}$ is the radius in the middle of the solenoid of a field line which grazes a bounding structure prior to reaching a gap and $r_{b}$ is the beam radius. As it turns out, from field line plots generated by Jin-Young Jung, this "scrape-off radius" is about $2.77 \mathrm{~cm}$, larger than the beam radius of $2.6 \mathrm{~cm}$. So there will be insignificant scrape-off of the beam when on-center. We might then consider the effect of displacement of the center of the beam. Taking a nominal maximum displacement of $0.5 \mathrm{~cm}$, the fractional overlap of the displaced beam and the scrape-off radius is less than the fractional displacement, i.e. less than 19\%. Considering that the average displacement is most likely less than this, and considering other uncertainties in the calculation, we deem loss due to electrons born from ionization on field lines that intersect walls to be a negligible effect and so take $f_{b}=1$.

Summarizing the results of the preceding two paragraphs, if the total number of electrons produced by ionization per beam pulse is $N_{e}$, the number that get accelerated through an active gap is

$$
N_{e, a c c e l} \approx f_{p} f_{b} N_{e}
$$

with $f_{p} \sim 0.1$ and $f_{b} \sim 1$.

The number of ionization-produced electrons per beam ion is given by

$$
N_{e} / N_{b}=\int n_{g} \sigma_{\text {ionize }} d z
$$

A simple formula for $\sigma_{\text {ionize }}$ for fully stripped ions is given in Ref. [4]. Our beam ions, however, are not fully stripped. Ionizing collisions with large impact parameter (greater than about the atomic radius) see a shielded nucleus while those at significantly smaller impact parameter see the full nuclear charge. Hence the total cross section is a blend of the fully stripped cross section evaluated with the full nuclear charge and the cross section evaluated for charge 1 [3]. We take here, as our estimate, the mean of these values. This 


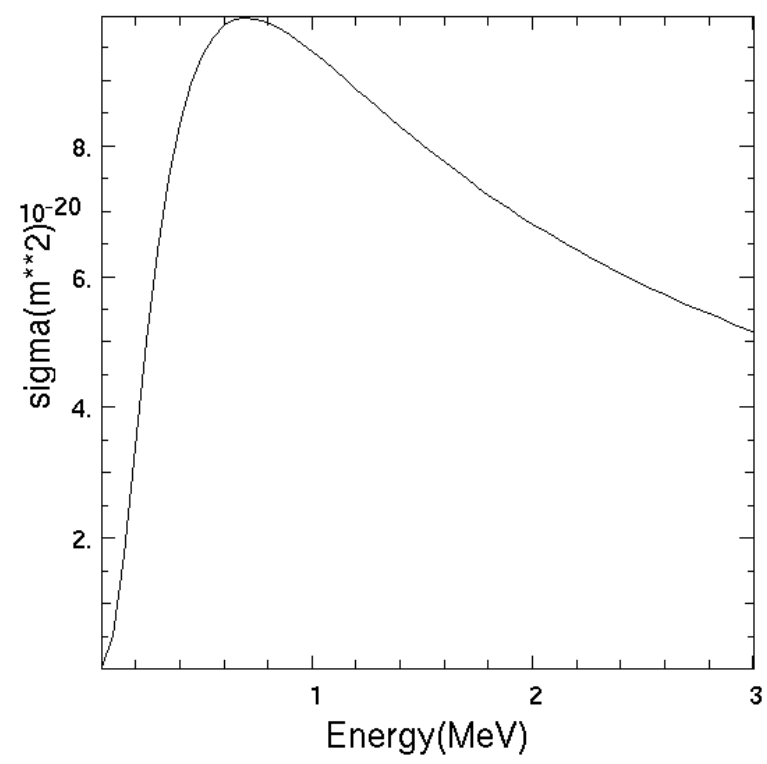

Figure 3: Waveform for an NCX-II accelerating module

mean is shown as a function of energy in Fig. 3. From that figure we infer that we can obtain a conservative upper-bound estimate of $N_{e}$ by taking $\sigma=$ const $=8 \times 10^{-20} \mathrm{~m}^{2}$. Noting that $n_{g}$ can be expressed in terms of the background pressure in Torr at room temperature as $n_{g}=3.5 \times 10^{22} P($ torr $)$ molecules $/ \mathrm{m}^{-} 3=7 \times 10^{22} \mathrm{P}($ torr $)$ atoms $/ \mathrm{m}^{3}$ (we assume here molecular nitrogen as the background gas), we estimate the number of electrons produced in the accelerator of length $L$ that see a live accelerating gap to be

$N_{e, a c c e l} / N_{b}=f_{p} f_{b} n_{g} \sigma L=0.1 \times 7 \times 10^{22} \times 8 \times 10^{-20} \times L(m) \times P($ torr $)=5600 P($ torr $) \times(L / 10 \mathrm{~m})$

The total number of beam ions per pulse is that of 10 Amps over $1 \mathrm{~ns}$, or $6 \times 10^{10}$. Hence the total number of energetic electrons produced per pulse is

$$
N_{e, \text { accel }} \sim 3.4 \times 10^{14} \mathrm{P}(\text { torr })(L / 10 \mathrm{~m})
$$

and the total energy in these electrons (accelerated to an energy of at most about $375 \mathrm{KeV}$ ) is

$$
E_{e} \sim 1.3 \times 10^{20} P(\text { torr })(L / 10 m) \mathrm{eV} \sim 21 P(\text { torr })(L / 10 \mathrm{~m}) \text { Joule }
$$

The NRL tables contain a formula for x-ray production by stopped electrons, namely x-ray power/beam power $=7 \times 10^{-4} Z E_{e}(\mathrm{MeV})$, where $Z$ is the average atomic number of atoms in the target. Taking $Z=20$ and beam energy of $375 \mathrm{KeV}$ gives the average energy per incident electron as about $2 \mathrm{KeV}$, and the total energy per pulse in $\mathrm{x}$ rays as about

$$
E_{x} \sim 0.11 P(\text { torr })(L / 10 m) \text { Joule }
$$

The average-energy $(2 \mathrm{KeV})$ x-rays are easily stopped by the accelerator structure, but there could be some concern around the energetic tail of electrons. As a pessimistic limit we 
might ask what would be the dose in rads if all of the x-ray energy just calculated struck a $70 K G$ person (a highly unlikely occurrence); we find

$$
\text { Dose }=0.11 P(\text { torr })(L / 10 m) / 0.7=0.16 P(\text { torr })(L / 10 m) \operatorname{Rad}
$$

i.e. still rather small at the expected vacuums for NDCX-II.

\section{Effect of Ionization and Charge Exchange on the Beam Ions}

Ionization results in a loss of energy of the beam ion; the related process of charge exchange leads to a change of charge state of the beam ion, which would most likely result in that ion being lost from the beam (or at least lost from the focussed part of the beam). The fractional energy loss per beam ion from ionization is

$$
\Delta E_{\text {ioniz }} / E_{\text {beam }} \sim\left(N_{e} / N_{b}\right) I_{n l} / E_{\text {beam }}
$$

where $I_{n l} \cong 14.5 \mathrm{eV}$ is the ionization potential (here, evaluated for Nitrogen). Taking $N_{e}=$ $N_{e, \text { accel }} / f_{p} f_{b}$ from Eq. (4), we find

$$
\Delta E_{\text {ioniz }} / E_{\text {beam }} \sim 0.37 P(\text { torr })(L / 10 \mathrm{~m})
$$

and so is negligibly small for pressures in the microtorr range.

The charge exchange probability is given by

$$
P_{c x}=\int n_{g} \sigma_{c x} d z
$$

where $\sigma_{c x}$ is the charge exchange cross section. There are two charge exchange processes to consider, namely electron capture by beam ions and stripping of an electron from beam ions. From, for example, Fig 9 of Ref. [5], it can be seen that the capture cross section (which is what is called charge exchange in that reference) is quite small for ions in the several hundred keV/AMU range, in particular small compared to the ionization cross section. On the other hand the stripping cross section can be significant. Approximately, the stripping cross section is set [2] from the criterion that impact parameters less than the atomic radius of a typical background gas atom lead to stripping. For nitrogen gas, this gives us the estimate $\sigma_{c x}=2 \pi r_{A}^{2}$ where $r_{a}$ is the atomic radius and the extra factor of two comes from two atoms per molecule. Interestingly a quick google search shows a factor of almost two spread in quoted atomic radius for nitrogen. The values average around $70 \mathrm{pm}\left(7 \times 10^{-11} \mathrm{~m}\right)$. From this value we obtain the estimate $\sigma_{c x} \sim 3 \times 10^{-20} \mathrm{~cm}^{2}$, which is of the same order as, but smaller than, the ionization cross section. Estimating $n_{g}$ in molecules $/ \mathrm{m}^{3}$ as above, we find

$$
P_{c x} \sim 1 \times 10^{3} P(\text { torr })(L / 10 m)
$$

So even for a pressure of $10^{-5}$ Torr we would lose only about $1 \%$ of the beam to charge exchange. 
This work was performed under the auspices of the USDOE by LLNL under Contract DEAC52-07NA27344. The author thanks Igor Kaganovich for helpful discussions and emails.

\section{References}

[1] C.A.F. Pintao, R. Hessel, 'Secondary-electron yield of metals measured by a dynamic method", J. App. Physics 88, 478 (2000).

[2] A. Friedman, J.J. Barnard, R.H. Cohen, et al., "Beam dynamics of the Neutralized Drift Compression Experiment-II (NDCX-II), a novel pulse-compressing ion accelerator", submitted to Phys. Plasmas (2009).

[3] I.D. Kaganovich, private communication

[4] I.D. Kaganovich, E.A. Startsev, R.C.Davidson, "Scaling cross sections for ion-atom impact ionization", Phys. Plasmas 11, 1229 (2004).

[5] I.D. Kaganovich, A. Shnidman, H. Bebane, R.C. Davidson, "Calculation of chargechanging cross sections of ions or atoms colliding with fast ions using the classical trajectory method", NIM-A 606, 196 (2009). 